\title{
Cerebrovascular autoregulation in preterm fetal growth restricted neonates
}

\author{
Emily Cohen, ${ }^{1,2}$ Willem Baerts, ${ }^{1}$ Alexander Caicedo Dorado, ${ }^{3}$ Gunnar Naulaers, ${ }^{4}$ \\ Frank van Bel, ${ }^{1}$ Petra M A Lemmers ${ }^{1}$
}

'Department of Neonatology, Wilhelmina Children's Hospital/University Medical Centre Utrecht, Utrecht, The Netherlands

${ }^{2}$ The Ritchie Centre, Hudson Institute of Medical Research and Department of Paediatrics, Monash University, Melbourne, Victoria, Australia

${ }^{3}$ Department of Applied Mathematics and Computer Science, Faculty of Natural Sciences and Mathematics, Universidad del Rosario, Bogota, Colombia

${ }^{4}$ Department of Neonatology, University Hospital Leuven, Leuven, Belgium

Correspondence to Dr Petra M A Lemmers, Department of Neonatology, Wilhelmina Children's Hospital/ University Medical Centre Utrecht, Utrecht 3584AE, The Netherlands; p.lemmers@ umcutrecht.nl

Received 11 July 2017 Revised 12 September 2018 Accepted 22 September 2018
Check for updates

(C) Author(s) (or their employer(s)) 2018. No commercial re-use. See rights and permissions. Published by BMJ.

To cite: Cohen E, Baerts W, Caicedo Dorado A, et al. Arch Dis Child Fetal Neonatal Ed Epub ahead of print: [please include Day Month Year]. doi:10.1136/

archdischild-2017-313712

\section{ABSTRACT}

Objective To investigate the effect of fetal growth restriction (FGR) on cerebrovascular autoregulation in preterm neonates during the first 3 days of life.

Design Case-control study.

Setting Neonatal intensive care unit of the Wilhelmina Children's Hospital, The Netherlands.

Patients 57 FGR (birth weight $<10$ th percentile) and 57 appropriate for gestational age (AGA) (birth weight 20th-80th percentiles) preterm neonates, matched for gender, gestational age, respiratory and blood pressure support.

Methods The correlation between continuously measured mean arterial blood pressure and regional cerebral oxygen saturation was calculated to generate the cerebral oximetry index (COx). Mean COx was calculated for each patient for each postnatal day. The percentage of time with impaired autoregulation (COx>0.5) was also calculated.

Results FGR neonates had higher mean COx values than their AGA peers on day $2(0.15(95 \% \mathrm{Cl} 0.11$ to $0.18)$ vs 0.09 (95\% Cl 0.06 to 0.13$), p=0.029)$ and day $3(0.17(95 \% \mathrm{Cl} 0.13$ to 0.20$)$ vs $0.09(95 \% \mathrm{Cl} 0.06$ to $0.12), p=0.003)$ of life. FGR neonates spent more time with impaired autoregulation (COx value $>0.5)$ than controls on postnatal day $2(19 \%(95 \% \mathrm{Cl} 16 \%$ to $22 \%)$ vs $14 \%(95 \% \mathrm{Cl} 12 \%$ to $17 \%), \mathrm{p}=0.035)$ and day 3 (20\% (95\% Cl $17 \%$ to $24 \%)$ vs $15 \%$ (95\% Cl $12 \%$ to $18 \%), p=0.016)$.

Conclusion FGR preterm neonates more frequently display impaired cerebrovascular autoregulation compared with AGA peers on days 2 and 3 of life which may predispose them to brain injury. Further studies are required to investigate whether this impairment persists beyond the first few days of life and whether this impairment is linked to poor neurodevelopmental outcome.

\section{INTRODUCTION}

Cerebrovascular autoregulation is the ability to maintain stable cerebral perfusion despite fluctuations in arterial blood pressure. ${ }^{1}$ The cerebral arterioles constrict as blood pressure increases to prevent hyperperfusion of the brain. A decrease in blood pressure is compensated for by dilation of the cerebral arterioles to prevent hypoperfusion. ${ }^{1}$ Previous studies have indicated that cerebrovascular autoregulation may be dysfunctional in (sick) preterm neonates, predisposing them to haemorrhagic and ischaemic brain injury and neonatal death. ${ }^{2-6}$

Compared with preterm appropriate for gestational age (AGA) neonates, preterm fetal growth

\section{What is already known on this topic?}

- Cerebrovascular autoregulation is the ability to maintain stable cerebral blood flow despite fluctuations in arterial blood pressure.

- Cerebrovascular autoregulation can be impaired in preterm appropriate for gestational age (AGA) neonates, which predisposes these neonates to neurological injury.

\section{What this study adds?}

- Fetal growth restricted (FGR) preterm neonates more frequently display impaired cerebrovascular autoregulation compared with AGA peers on days 2 and 3 of life.

- Further research is needed to investigate whether this impairment persists and whether this impairment increases the risk of neurological injury in preterm FGR neonates.

restricted (FGR) neonates demonstrate higher cerebral oxygenation throughout the first few postnatal days. $^{78}$ This increase most likely reflects increased cerebral blood flow related to the brain-sparing phenomenon. Chronic fetal hypoxia and FGR have also been associated with cerebrovascular remodelling and altered cerebrovascular reactivity. ${ }^{910}$ These alterations in structural and functional properties of the cerebral vasculature may further impair cerebrovascular autoregulation and increase vulnerability of the brain to hypoperfusion and hyperperfusion within this subgroup of preterm neonates. To date, no studies have investigated cerebrovascular autoregulation in preterm FGR neonates. In this study, we thus aimed to investigate the effect of FGR on cerebrovascular autoregulation in preterm neonates throughout the first 3 days of life. We hypothesised that FGR would exacerbate the unfavourable effect of preterm birth on cerebrovascular autoregulation.

\section{PATIENTS AND METHODS}

\section{Patients and data collection}

We performed a retrospective case-control study on data from a large prospective cohort study investigating normative cerebral oxygen saturation $\left(\mathrm{rScO}_{2}\right)$ and cerebral fractional tissue oxygen extraction (cFTOE) values of prematurely born neonates (gestational age (GA) $<32$ weeks) admitted to the neonatal intensive care unit of the Wilhelmina 


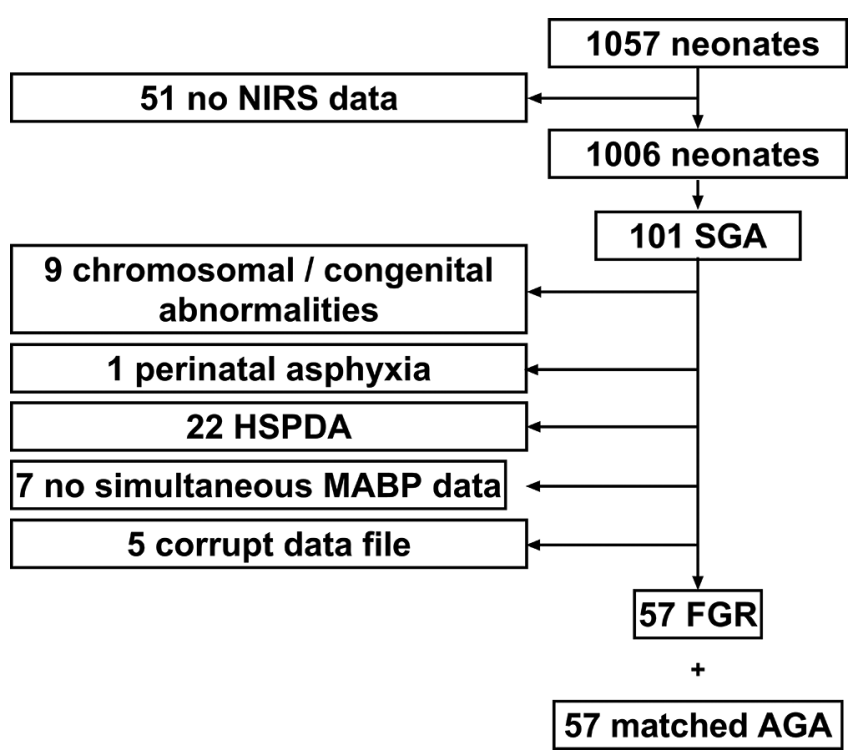

Figure 1 Patient selection. AGA, appropriate for gestational age; FGR, fetal growth restriction; HSPDA, haemodynamically significant persistent ductus arteriosus; MABP, mean arterial blood pressure; NIRS, nearinfrared spectroscopy; SGA, small for gestational age.

Children's Hospital between 2000 and 2013. ${ }^{11}$ In this observational study $\mathrm{rScO}_{2}$, was recorded by means of near-infrared spectroscopy during the first 3 days of life (INVOS 5100C with a small adult sensor, Covidien/Medtronic, Boulder, Colorado, USA). cFTOE was calculated as $\left(\mathrm{SpO}_{2}-\mathrm{rScO}_{2}\right) / \mathrm{SpO}_{2}$. In addition, physiological variables used for routine clinical monitoring were recorded. These data included continuous measurement of mean arterial blood pressure (MABP) by an indwelling arterial catheter and arterial oxygen saturation by pulse oximetry $\left(\mathrm{SpO}_{2}\right)$. Clinical data included a daily blood pressure support score adapted from Krediet $e t a l^{12}$ and a respiratory support score classified as non-invasive (spontaneously breathing with low flow or continuous/bilevel positive airway pressure) or invasive (synchronised intermittent mandatory ventilation or high-frequency oscillatory ventilation).

From the database, we identified all FGR neonates-defined as by having a birth weight below the 10th percentile for GA on the Fenton growth curve ${ }^{13}$ - for whom simultaneous $\mathrm{rScO}_{2}$ and MABP data were available (figure 1). Each FGR neonate was matched with an AGA control (birth weight between the 20th and 80th percentiles). Matching criteria were gender, GA ( \pm 4 days) and similar blood pressure and respiratory support status. Neonates with chromosomal abnormalities, congenital abnormalities influencing the systemic and/or cerebral circulation (including congenital heart disease), confirmed haemodynamically significant persistent ductus arteriosus on echocardiography, ${ }^{14}$ proven perinatal sepsis and perinatal asphyxia ${ }^{15}$ were excluded. For eligible infants, we collected information regarding the last fetal Doppler measurements when available. A middle cerebral artery pulsatility index below the fifth percentile or a placental-cerebral ratio above 1.1 was considered abnormal and a sign of prenatal brain sparing.

\section{Autoregulation analysis}

Data were analysed using in-house developed software (SignalBase, University Medical Centre Utrecht, The Netherlands). To estimate cerebrovascular autoregulation, the correlation between continuously measured MABP and $\mathrm{rScO}_{2}$ was used. ${ }^{16}$ Data were visually inspected in 30 min epochs. Data that included MABP,
$\mathrm{rScO}_{2}$ and/or $\mathrm{SpO}_{2}$ artefacts were excluded from further analysis. Due to the fact that $\mathrm{SpO}_{2}$ fluctuations influence $\mathrm{rScO}_{2}$ irrespective of MABP changes, we excluded any $\mathrm{SpO}_{2}$ fluctuations exceeding $10 \%$ compared with the epoch's baseline. ${ }^{17}$ It is estimated that the $\mathrm{rScO}_{2}$ response to an MABP change occurs with a delay of approximately $10 \mathrm{~s}$ in neonates. ${ }^{18}$ To take this physiological delay into account, the MABP signal was subjected to a preprocessing time shift of $+10 \mathrm{~s}^{19}{ }^{19} \mathrm{MABP}$ and $\mathrm{rScO}_{2}$ signals were filtered and subsequently down-sampled at $0.1 \mathrm{~Hz}$ to eliminate high-frequency noise. ${ }^{20}$ A Pearson's correlation coefficient was calculated between 30 paired samples of MABP and $\mathrm{rScO}_{2}$, generating the cerebral oximetry index (COx) ${ }^{20}{ }^{21}$ The COx was updated every $10 \mathrm{~s}$ using a moving $300 \mathrm{~s}$ window $(97 \%$ overlap)..$^{20}$ The average MABP and the coefficient of variation (CV) of MABP were also calculated for each $300 \mathrm{~s}$ window. The MABP CV can be used as a measure of blood pressure variability $^{22} 23$ and blood pressure variability has been shown to affect cerebrovascular autoregulation. ${ }^{24}$

Mean COx was calculated for each patient for each postnatal day. A COx value $>0.5$ was considered to represent impaired cerebrovascular autoregulation. ${ }^{20}$ The percentage of time spent with a $\mathrm{COx}>0.5$ was also calculated for each patient for each postnatal day. Cerebrovascular autoregulation was further investigated relative to the neonate's MABP. ${ }^{20} \mathrm{COx}$ values were grouped and averaged in $5 \mathrm{~mm} \mathrm{Hg}$ bins, where MABP was corrected for GA (MABP-GA in completed weeks). ${ }^{21} 25$ The mean $\mathrm{COx}$ and the time spent with a $\mathrm{COx}>0.5$ were calculated for each MABP bin in each patient.

\section{Statistical analysis}

Data were statistically analysed using SPSS for Windows V.22 (IBM, Released 2013). To analyse demographic and clinical variables, $\mathrm{X}^{2}$ test, Fisher's exact test or Mann-Whitney $\mathrm{U}$ test was used, as appropriate. These data are represented as $\mathrm{n}(\%)$ or median (range).

Physiological variables $\left(\mathrm{rScO}_{2}\right.$, cFTOE, MABP and $\left.\mathrm{SpO}_{2}\right)$, MABP CV and cerebrovascular autoregulation variables were analysed using a mixed model approach to explore the effect of postnatal age and group. These analyses were corrected for GA. A mixed model was also used to explore the effect of GA-corrected MABP bin and group on cerebrovascular autoregulation variables. Statistically significant overall group differences were followed up with single analysis of (co)variance to explore on which postnatal day, respectively, in which MABP bin, group differences occurred. These data are represented as mean with bootstrapped 95\% CIs.

\section{RESULTS}

For a total of 57 (61\% male) FGR neonates simultaneous and artefact-free MABP and $\mathrm{rScO}_{2}$ data were available for cerebrovascular autoregulation analysis. Demographic and clinical data are presented in table 1. FGR pregnancies were more often associated with pre-eclampsia/Hemolysis Elevated Liver enzymes and Low Platelets (HELLP) and FGR fetuses were more frequently delivered by caesarean section. Prenatal Doppler measurements were available for $79 \%$ of the FGR fetuses. Of these, 91\% showed signs of brain sparing. For the AGA population this information was lacking in the majority of cases (88\%). There were no differences between the FGR and AGA group for antenatal corticosteroid administration or GA at birth. FGR neonates were born with a significantly lower birth weight and they had a higher lactate than AGA controls. Apgar score at 1 min was similar between the groups, however FGR neonates had slightly higher Apgar score at $5 \mathrm{~min}$. There were no 
Table 1 Demographics and clinical data

\begin{tabular}{|c|c|c|c|}
\hline & FGR & AGA & $P$ values \\
\hline $\mathrm{n}$ (\% male) & $57(61)$ & $57(61)$ & NA \\
\hline Birth weight, $\mathrm{g}$ & $840(515-1235)$ & $1345(840-1940)$ & $<0.001$ \\
\hline Birth weight percentile & $6(1-10)$ & $54(21-79)$ & $<0.001$ \\
\hline Head circumference, $\mathrm{cm}$ & $25(20.5-27.3)$ & $27.5(23.2-31.0)$ & $<0.001$ \\
\hline Head circumference percentile & $6(0-63)$ & $52(11-99)$ & $<0.001$ \\
\hline Gestational age, weeks & $30\left(26^{4} / 7-32\right)$ & $30 I_{7}\left(26^{4} I_{7}-31 \%_{7}\right)$ & 0.847 \\
\hline Pre-eclampsia/HELLP & $31(54 \%)$ & $6(11 \%)$ & $<0.001$ \\
\hline Doppler data available & $45(79 \%)$ & $7(12 \%)$ & $<0.001$ \\
\hline Prenatal brain-sparing (MCA-PI or PCR abnormal) & $41(91 \%)$ & $2(29 \%)$ & 0.001 \\
\hline Antenatal corticosteroids & $46(81 \%)$ & $45(79 \%)$ & 0.815 \\
\hline Delivery by caesarean section & $55(97 \%)$ & $29(51 \%)$ & $<0.001$ \\
\hline Apgar score at $1 \mathrm{~min}$ & $7(1-10)$ & $7(0-10)$ & 0.722 \\
\hline Apgar score at $5 \mathrm{~min}$ & $9(6-10)$ & $8(4-10)$ & 0.031 \\
\hline Lactate, $\mathrm{mmol} / \mathrm{L}$ & $5.5(2.0-18.1)$ & $3.8(1.2-26.10)$ & $<0.001$ \\
\hline Umbilical artery $\mathrm{pH}$ & $7.25(6.95-7.37)$ & $7.26(7.06-7.41)$ & 0.083 \\
\hline Predominant form of respiratory support & & & 0.663 \\
\hline Low flow/CPAP/BIPAP & $44(77 \%)$ & $42(74 \%)$ & \\
\hline SIMV/HFOV & $13(23 \%)$ & $15(26 \%)$ & \\
\hline Predominant form of blood pressure support & & & 0.802 \\
\hline No support & $47(83 \%)$ & $49(86 \%)$ & \\
\hline Mild support & $6(10 \%)$ & $4(7 \%)$ & \\
\hline Moderate to intensive support & $4(7 \%)$ & $4(7 \%)$ & \\
\hline IVH & $9(16 \%)$ & $10(18 \%)$ & 1 \\
\hline Grade I & 7 & 4 & \\
\hline Grade II & 3 & 5 & \\
\hline Grade III & 0 & 0 & \\
\hline Grade IV & 0 & 0 & \\
\hline \multicolumn{4}{|l|}{ Data available for autoregulation analysis, hour } \\
\hline Overall & $44.8(2.1-64.0)$ & $49.2(19.7-62.2)$ & 0.066 \\
\hline Day 1 & $13.65(0.3-20.3)$ & $14.5(1.3-20.9)$ & 0.528 \\
\hline Day 2 & $17.18(0.6-22.4)$ & $18.4(6.0-21.7)$ & 0.083 \\
\hline Day 3 & $16.9(4.0-22.2)$ & $17.6(4.2-22.1)$ & 0.88 \\
\hline
\end{tabular}

Data are presented as median (range) or $n(\%)$.

AGA, appropriate for gestational age; BIPAP, bilevel positive airway pressure; CPAP, continuous positive airway pressure; FGR, fetal growth restriction; HELLP, Hemolysis Elevated Liver enzymes and Low Platelets; HFOV, high-frequency oscillatory ventilation; IVH, intraventricular haemorrhage; MCA-PI, middle cerebral artery pulsatility index; NA, not applicable; PCR, placental-cerebral ratio; SIMV, synchronised intermittent mandatory ventilation.

differences between the groups regarding respiratory and blood pressure support scores.

\section{Cerebrovascular autoregulation}

Cerebrovascular autoregulation results are shown in figures 2 and 3 .
A

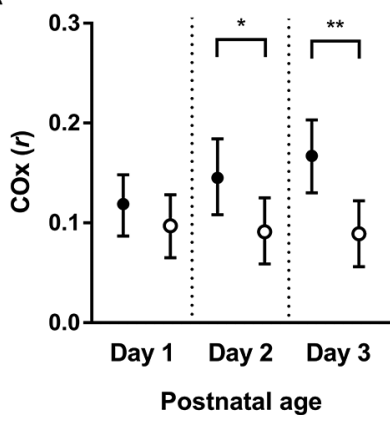

B

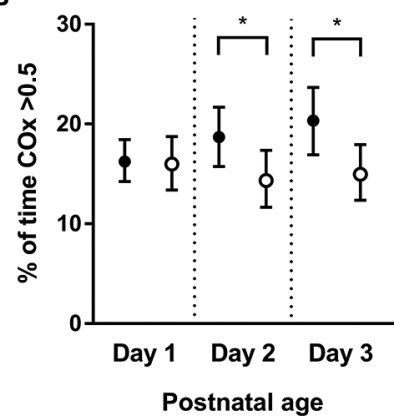

Figure 2 Mean $\mathrm{COx}(\mathrm{A})$ and time spent with impaired autoregulation (B). Fetal growth restricted (FGR) neonates are represented by the closed circles and appropriate for gestational age (AGA) neonates are represented by the open circles. Error bars represent bootstrapped $95 \%$ Cls. ${ }^{*} \mathrm{P}<0.05 ;{ }^{*} \mathrm{P}<0.01$. COx, cerebral oximetry index.
Postnatal age had no significant effect on cerebrovascular autoregulation variables, yet a significant group effect was found for these variables. Further testing revealed that FGR neonates had higher COx values than their AGA peers on day $2(\mathrm{p}=0.029, \mathrm{r}=0.20)$ and day $3(\mathrm{p}=0.003, \mathrm{r}=0.28)($ figure $1 \mathrm{~A})$ and FGR neonates also spent more time with a COx value $>0.5$ than controls on postnatal day $2(\mathrm{p}=0.035, \mathrm{r}=0.20)$ and day 3 $(\mathrm{p}=0.016, \mathrm{r}=0.23)$ (figure 1B).

A significant group effect was also demonstrated when cerebrovascular autoregulation variables were examined in relation to GA-adjusted MABP bins. FGR neonates had significantly higher $\mathrm{COx}$ values and spent significantly more time with a COx value $>0.5$ at the $10-15 \mathrm{~mm} \mathrm{Hg}(\mathrm{COx}: \mathrm{p}=0.006$, $\mathrm{r}=0.26, \mathrm{COx}>0.5: \mathrm{p}=0.008, \mathrm{r}=0.25)$ and $15-20 \mathrm{~mm} \mathrm{Hg}$ bins (COx: $\mathrm{p}=0.004, \mathrm{r}=0.28, \mathrm{COx}>0.5: \mathrm{p}=0.006, \mathrm{r}=0.27$ ) (figure 2).

\section{Physiological variables and blood pressure variability}

Physiological variables and blood pressure variability are shown in table 2. $\mathrm{rScO}_{2}$ was higher and cFTOE was lower in FGR compared with AGA neonates, reaching statistical significance on day 2 and day 3. $\mathrm{SpO}_{2}$ was comparable between the groups. MABP and MABP CV increased significantly with advancing 
A

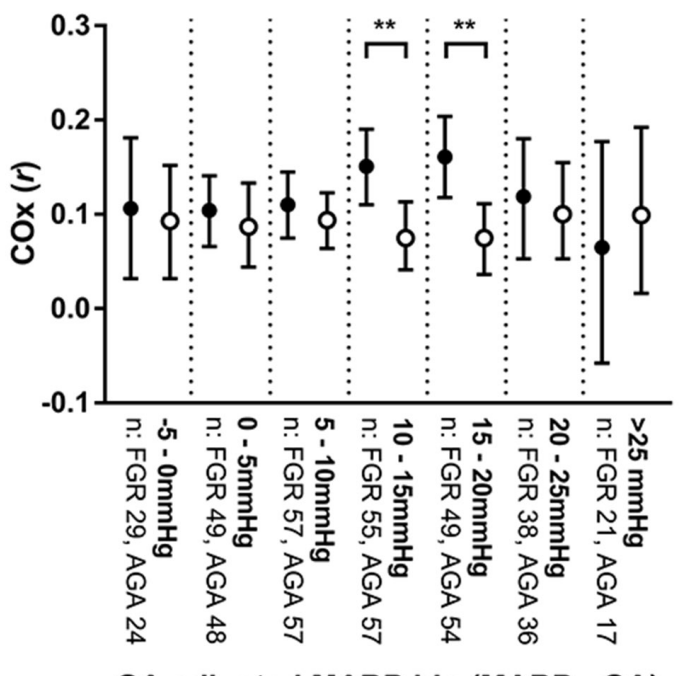

B

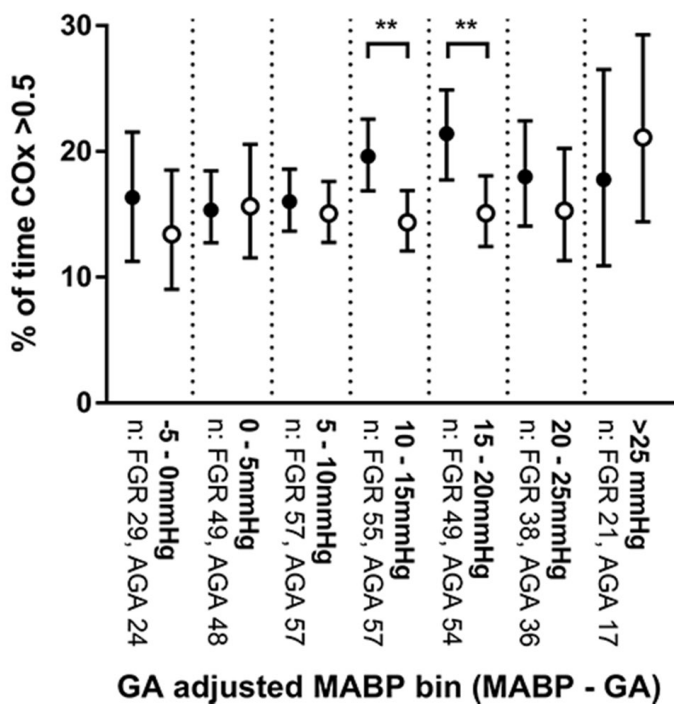

Figure 3 Mean $\mathrm{COx}(\mathrm{A})$ and time spent with impaired autoregulation (B) for each gestational age-adjusted MABP bin. FGR neonates are represented by the closed circles and AGA neonates are represented by the open circles. Error bars represent bootstrapped $95 \%$ Cls. ${ }^{*} \mathrm{P}<0.01$. $A G A$, appropriate for gestational age; COx, cerebral oximetry index; FGR, fetal growth restriction; GA, gestational age; MABP, mean arterial blood pressure.

postnatal age, however no group differences were detected for these variables.

\section{DISCUSSION}

To our knowledge, this is the first study to investigate the effect of FGR on cerebrovascular autoregulation in preterm neonates throughout the first postnatal days. This study uses a convenience sample based on data from an existing cohort study. ${ }^{11}$ Infants with FGR were considered cases, and appropriately

\begin{tabular}{|c|c|c|c|}
\hline & FGR & AGA & $P$ values \\
\hline \multicolumn{4}{|l|}{$\mathrm{rScO}_{2}, \%$} \\
\hline Postnatal day 1 & 71 (69 to 73 ) & 69 (68 to 71) & 0.311 \\
\hline Postnatal day 2 & 74 (72 to 75$)$ & 69 (68 to 71$)$ & 0.002 \\
\hline Postnatal day 3 & 71 (69 to 73) & 69 (67 to 70) & 0.042 \\
\hline \multicolumn{4}{|l|}{ cFTOE } \\
\hline Postnatal day 1 & $0.26(0.24$ to 0.29$)$ & $0.29(0.27$ to -0.31$)$ & 0.152 \\
\hline Postnatal day 2 & $0.23(0.22$ to 0.25$)$ & 0.29 (0.26 to 0.31$)$ & 0.001 \\
\hline Postnatal day 3 & $0.26(0.25$ to 0.28$)$ & 0.30 (0.27 to 0.33$)$ & 0.032 \\
\hline \multicolumn{4}{|l|}{$\mathrm{SpO}_{2}$} \\
\hline Postnatal day 1 & 96 (95 to 96$)$ & 96 (95 to 96) & 0.399 \\
\hline Postnatal day 2 & 96 (96 to 97) & 96 (95 to 96) & 0.404 \\
\hline Postnatal day 3 & 96 (96 to 97) & 96 (95 to 97) & 0.215 \\
\hline \multicolumn{4}{|l|}{ MABP } \\
\hline Postnatal day $1^{*}$ & $37(36$ to 38$)$ & 38 (37 to 39$)$ & 0.164 \\
\hline Postnatal day $2 \dagger$ & 41 (40 to 43 ) & 40 (39 to 42 ) & 0.471 \\
\hline Postnatal day 3 & 43 (41 to 45$)$ & 42 (41 to 44$)$ & 0.461 \\
\hline \multicolumn{4}{|l|}{ MABP CV } \\
\hline Postnatal day $1 \dagger$ & 3.8 (3.6 to 4.1$)$ & 4.1 (3.9 to 4.3$)$ & 0.179 \\
\hline Postnatal day $2 \ddagger$ & 4.0 (3.8 to 4.2 ) & 4.1 (3.9 to 4.4$)$ & 0.539 \\
\hline Postnatal day 3 & 4.3 (4.1 to 4.6$)$ & $4.4(4.2$ to 4.6$)$ & 0.609 \\
\hline
\end{tabular}

Data are presented as mean (bootstrapped $95 \% \mathrm{Cl}$ ). All variables were corrected for gestational age.

*Versus day $3 p<0.001$

tVersus day $3 p<0.01$.

$\ddagger$ Versus day $3 p<0.05$.

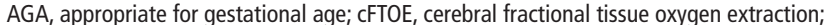

CV, coefficient of variation; FGR, fetal growth restriction; MABP, mean arterial blood pressure. matched controls were selected from the same cohort. With this sample we were able to demonstrate a significant difference in cerebrovascular autoregulation between the two groups. FGR neonates showed higher mean COx values than their AGA peers. Although mean COx did not exceed the threshold of impaired cerebrovascular autoregulation, FGR neonates did spend significantly more time with impaired cerebrovascular autoregulation $(\mathrm{COx}>0.5)$ than AGA neonates on days 2 and 3 of life.

In developed countries, FGR is most commonly caused by placental insufficiency leading to chronic fetal hypoxia. ${ }^{26}$ Studies performed in adults at high altitude have established that chronic hypoxia significantly impairs cerebrovascular autoregulation. ${ }^{27-29}$ Although these changes appear to be functional and reversible after reoxygenation, ${ }^{29}$ exposure to chronic hypoxia during a critical window of brain development may more permanently alter cerebrovascular structure and function resulting in lasting impairments in cerebrovascular autoregulation. In fact, animal studies have demonstrated that chronic fetal hypoxia promotes cerebrovascular remodelling, resulting in altered arterial wall structure and function. ${ }^{30-32}$ Although it has been hypothesised that some of these alterations aim to preserve energy while still maintaining basic contractile function during exposure to chronic intrauterine hypoxia, ${ }^{31}$ the consequences on postnatal cerebrovascular function are unknown. A recent study demonstrated significant structural changes within the cerebral vasculature of FGR lambs, including reduced endothelial proliferation, ${ }^{32}$ which may contribute to pressure passivity. ${ }^{33}$ These authors also reported increased permeability of the blood-brain barrier and a higher incidence of haemorrhages in their FGR lambs. ${ }^{32}$ Moreover, Soul et al reported that human neonates born from pregnancies complicated by pregnancy-induced hypertension or placental infarction spent more time with impaired cerebrovascular autoregulation than neonates born from pregnancies without these complications. ${ }^{23}$ Although these authors did not aim to investigate the effect of FGR, it is plausible that these pregnancy complications were associated with placental insufficiency resulting in chronic fetal hypoxia and FGR. 
It is likely that the altered cerebrovascular reactivity of our FGR neonates is related to adaptations of the cerebral vasculature to the unfavourable prenatal environment. Surprisingly, however, cerebrovascular dysfunction only became apparent from day 2 of life. Our method to investigate cerebrovascular autoregulation requires spontaneous fluctuations in blood pressure. Dysfunctional autoregulation may only become detectable when there is sufficient blood pressure variability. ${ }^{23}{ }^{24} \mathrm{In}$ our study, blood pressure variability increased significantly with advancing postnatal age, which may explain why dysfunctional autoregulation became apparent from day 2 of life.

Cerebrovascular autoregulation is believed to have limited capacity. Within a specific range of blood pressures, the cerebral vasculature is able to maintain stable cerebral perfusion. This is referred to as the autoregulatory plateau. ${ }^{1}$ When blood pressure falls below a lower threshold, cerebral blood flow will decrease. Similarly, when blood pressure exceeds an upper threshold, cerebral blood flow will increase. ${ }^{1}$ Most of the preterm AGA studies focus on the effect of hypotension on cerebrovascular autoregulation. Gilmore et al suggested that dysfunctional autoregulation was more likely to occur during hypotensive rather than hypertensive episodes. ${ }^{20}$ However, the autoregulatory ability of our AGA neonates did not vary over the range of blood pressures studied. Cerebrovascular autoregulation of FGR neonates, on the other hand, appeared to mainly be affected at mid to high blood pressures. When relating these findings to the autoregulatory plateau theorem, the findings suggest dysfunctional cerebral vasoconstriction of the FGR neonates at high cerebral perfusion pressure, which may be related to long-standing cerebral vasodilation due to the brain-sparing effect. Smaller numbers (n) and large variation within the data at the extreme upper end of the blood pressure range may explain why no significant group differences occurred here. The fact that only a limited number of neonates were exposed to (relatively mild) hypotension may explain why we did not observe a relationship between cerebrovascular autoregulation and hypotension.

The method used in this study analyses cerebrovascular autoregulation in the time domain. This technique allows quantification of the relationship between blood pressure and cerebral oxygenation but does not specify at which frequency of blood pressure fluctuations cerebrovascular autoregulation may be impaired. Cerebrovascular autoregulation is believed to prevent transmission of slow MABP fluctuations to the cerebral circulation. ${ }^{20}$ By downsampling our data to $0.1 \mathrm{~Hz}$ we ensured that oscillations below $0.05 \mathrm{~Hz}$ were investigated (according to the Nyquist sampling theorem). ${ }^{20}$ Nevertheless, previous studies have indicated that prematurity affects cerebrovascular autoregulation predominantly in the ultralow frequencies $(0.003-$ $0.01 \mathrm{~Hz}) .{ }^{424}$ Analysis in the frequency domain, which enables the distinction between low and ultralow frequencies, may thus provide additional information. Moreover, cerebrovascular autoregulation may depend on the magnitude of blood pressure changes, which can be incorporated in frequency domain analysis. ${ }^{24}$ Also, it is plausible that the delay in $\mathrm{rScO}_{2}$ response following an MABP change varies between patients. Frequency domain analysis eliminates the effect of this delay. ${ }^{34}$ Future studies employing this technique to further investigate cerebrovascular autoregulation in preterm FGR neonates would be valuable. Nevertheless, analysis in the time domain has been found to be more robust. ${ }^{35}$

We acknowledge the limitations of this study. First, it is important to note that $\mathrm{rScO}_{2}$ can only accurately reflect fluctuations in cerebral blood flow (CBF) if arterial oxygenation and cerebral metabolic demand remain constant. Moreover, cerebrovascular reactivity is influenced by oxygen and carbon dioxide concentrations, metabolic demand and neuronal factors. ${ }^{1}$ We only analysed data with stable $\mathrm{SpO}_{2}$, to minimise the effect of $\mathrm{SpO}_{2}$ as a potential confounding factor. However, we were unable to correct for other potentially confounding factors, such as hypocapnia or hypercapnia and hypoglycaemia or hyperglycaemia. Actually, FGR neonates and infants can present with altered acid base status ${ }^{36}$ and sympathetic tone, ${ }^{3738}$ and they may have different cerebral metabolism. ${ }^{8}$ Moreover, there is evidence that vasoreactivity to some of these factors is altered in fetuses exposed to chronic hypoxia. ${ }^{30} 31$ Mechanistic studies exploring the interplay between these factors, vasoreactivity and cerebrovascular autoregulation in the FGR brain-and whether this differs from the AGA brain-would provide important information. Second, we cannot conclude whether the observed differences in cerebrovascular autoregulation between FGR and AGA neonates are of clinical importance. Our results showed a true, yet small to medium effect of FGR on cerebrovascular autoregulation. ${ }^{39}$ Previous studies have related impaired cerebrovascular autoregulation to ultrasonographic evidence of brain damage in AGA preterm neonates. ${ }^{4524} 40$ Our findings raise caution regarding the use of inotropic agents to significantly increase blood pressure in FGR neonates. Appropriately powered longitudinal studies investigating whether the observed differences in cerebrovascular autoregulation result in altered neurodevelopmental outcome would be very pertinent.

\section{CONCLUSIONS}

FGR neonates more frequently display impaired cerebrovascular autoregulation compared with AGA peers on days 2 and 3 of life. This may predispose them to brain injury. Longitudinal studies that extend beyond the first few days of life are warranted to investigate whether this impairment persists and whether it contributes to the increased risk of abnormal neurodevelopmental outcome of the FGR population. It is crucial that this topic is further investigated, as this will provide us with important insights to improve clinical care for FGR neonates.

Acknowledgements We thank René van de Vosse (University Medical Centre Utrecht) for developing the in-house software SignalBase that was used for data analysis. The authors are grateful for the suggestions of Associate Professor Flora Wong (Monash Newborn, Monash Health and The Ritchie Centre, Monash University, Australia) to improve this manuscript.

Contributors EC carried out all data analyses, interpreted and critically appraised the data and drafted the initial manuscript. FvB, WB and PMAL were responsible for data collection, contributed substantially to interpretation and critical appraisal of the data and critically reviewed the manuscript. ACD and GN contributed substantially to interpretation and critical appraisal of the data and critically reviewed the manuscript. All authors have seen and approved the final version of the manuscript as submitted.

Funding A scholarship was provided to EC by Red Nose Australia. ACD is a postdoctoral fellow of the Research Foundation Flanders (FWO).

Competing interests None declared.

Patient consent Not required.

Ethics approval Medical Ethical Committee of the University Medical Centre Utrecht.

Provenance and peer review Not commissioned; externally peer reviewed.

\section{REFERENCES}

1 Greisen G. Autoregulation of cerebral blood flow in newborn babies. Early Hum Dev 2005;81:423-8.

2 Wong FY, Leung TS, Austin T, et al. Impaired autoregulation in preterm infants identified by using spatially resolved spectroscopy. Pediatrics 2008;121:e604-11.

3 Mitra S, Czosnyka M, Smielewski P, et al. Heart rate passivity of cerebral tissue oxygenation is associated with predictors of poor outcome in preterm infants. Acta Paediatr 2014;103:e374-82. 
4 Tsuji M, Saul JP, du Plessis A, et al. Cerebral intravascular oxygenation correlates with mean arterial pressure in critically ill premature infants. Pediatrics 2000;106:625-32.

5 O'Leary H, Gregas MC, Limperopoulos C, et al. Elevated cerebral pressure passivity is associated with prematurity-related intracranial hemorrhage. Pediatrics 2009;124:302-9.

6 Rhee CJ, Fraser CD, Kibler K, et al. The ontogeny of cerebrovascular pressure autoregulation in premature infants. J Perinatol 2014;34:926-31.

7 Ishii H, Takami T, Fujioka T, et al. Comparison of changes in cerebral and systemic perfusion between appropriate- and small-for-gestational-age infants during the first three days after birth. Brain Dev 2014;36:380-7.

8 Cohen E, Baerts W, Alderliesten T, et al. Growth restriction and gender influence cerebral oxygenation in preterm neonates. Arch Dis Child Fetal Neonatal Ed 2016;101:F156-61.

9 Pearce WJ, Butler SM, Abrassart JM, et al. Fetal cerebral oxygenation: the homeostatic role of vascular adaptations to hypoxic stress. Adv Exp Med Biol 2011;701:225-32

10 Salihagić-Kadić A, Medić M, Jugović D, et al. Fetal cerebrovascular response to chronic hypoxia--implications for the prevention of brain damage. J Matern Fetal Neonatal Med 2006;19:387-96.

11 Alderliesten T, Dix L, Baerts W, et al. Reference values of regional cerebral oxygen saturation during the first 3 days of life in preterm neonates. Pediatr Res 2016;79:55-64.

12 Krediet TG, Valk L, Hempenius I, et al. Nitric oxide production and plasma cyclic guanosine monophosphate in premature infants with respiratory distress syndrome. Biol Neonate 2002:82:150-4.

13 Fenton TR. Fenton preterm growth chart: actual age calculator v6. http://www. ucalgary.ca/fenton/2013chart (accessed 11 Jul 2014)

14 Dix LM, Blok CA, Lemmers PM, et al. Early end-tidal carbon monoxide levels, patency of the ductus arteriosus and regional cerebral oxygenation in preterm infants. Neonatology 2014;105:161-5.

15 Anon. Executive summary: Neonatal encephalopathy and neurologic outcome, second edition. Report of the American College of Obstetricians and Gynecologists' Task Force on Neonatal Encephalopathy. Obstet Gynecol 2014;123:896-901.

16 Brady K, Joshi B, Zweifel C, et al. Real-time continuous monitoring of cerebral blood flow autoregulation using near-infrared spectroscopy in patients undergoing cardiopulmonary bypass. Stroke 2010;41:1951-6.

17 Caicedo A, Naulaers G, Wolf M, et al. Assessment of the myogenic and metabolic mechanism influence in cerebral autoregulation using near-infrared spectroscopy. Adv Exp Med Biol 2012;737:37-44.

18 Elwell CE, Cope M, Delpy DT. An analytical method for determining cerebrovascular transit time using near infrared spectroscopy. Adv Exp Med Biol 1997;428:561-8.

19 Caicedo A, Naulaers G, Lemmers P, et al. Detection of cerebral autoregulation by nearinfrared spectroscopy in neonates: performance analysis of measurement methods. J Biomed Opt 2012;17:117003.

20 Gilmore MM, Stone BS, Shepard JA, et al. Relationship between cerebrovascular dysautoregulation and arterial blood pressure in the premature infant. J Perinatol 2011;31:722-9.

21 Brady KM, Lee JK, Kibler KK, et al. Continuous time-domain analysis of cerebrovascular autoregulation using near-infrared spectroscopy. Stroke 2007;38:2818-25.
22 Parati G, Ochoa JE, Lombardi C, et al. Assessment and management of blood-pressure variability. Nat Rev Cardiol 2013;10:143-55.

23 Soul JS, Hammer PE, Tsuji M, et al. Fluctuating pressure-passivity is common in the cerebral circulation of sick premature infants. Pediatr Res 2007;61:467-73.

24 Wong FY, Silas R, Hew S, et al. Cerebral oxygenation is highly sensitive to blood pressure variability in sick preterm infants. PLoS One 2012;7:e43165.

25 Hahn GH, Maroun LL, Larsen N, et al. Cerebral autoregulation in the first day after preterm birth: no evidence of association with systemic inflammation. Pediatr Res 2012;71:253-60

26 Cox P, Marton T. Pathological assessment of intrauterine growth restriction. Best Pract Res Clin Obstet Gynaecol 2009;23:751-64.

27 Iwasaki K, Zhang R, Zuckerman JH, et al. Impaired dynamic cerebral autoregulation at extreme high altitude even after acclimatization. J Cereb Blood Flow Metab 2011;31:283-92.

28 Subudhi AW, Fan JL, Evero 0, et al. AltitudeOmics: cerebral autoregulation during ascent, acclimatization, and re-exposure to high altitude and its relation with acute mountain sickness. J App/ Physiol 2014;116:724-9.

29 Ainslie PN, Ogoh S, Burgess K, et al. Differential effects of acute hypoxia and high altitude on cerebral blood flow velocity and dynamic cerebral autoregulation: alterations with hyperoxia. J App/ Physiol 2008;104:490-8.

30 Longo LD, Pearce WJ. Fetal cerebrovascular acclimatization responses to high-altitude, long-term hypoxia: a model for prenatal programming of adult disease? Am J Physiol Regul Integr Comp Physiol 2005;288:R16-24.

31 Pearce W. Hypoxic regulation of the fetal cerebral circulation. J App/ Physio 2006;100:731-8

32 Castillo-Melendez M, Yawno T, Allison BJ, et al. Cerebrovascular adaptations to chronic hypoxia in the growth restricted lamb. Int J Dev Neurosci 2015;45:55-65.

33 Martínez-Orgado J, González R, Alonso MJ, et al. Endothelial factors and autoregulation during pressure changes in isolated newborn piglet cerebral arteries. Pediatr Res 1998;44:161-7.

34 Hahn GH, Heiring C, Pryds O, et al. Applicability of near-infrared spectroscopy to measure cerebral autoregulation noninvasively in neonates: a validation study in piglets. Pediatr Res 2011;70:166-70.

35 Eriksen VR, Hahn GH, Greisen G. Cerebral autoregulation in the preterm newborn using near-infrared spectroscopy: a comparison of time-domain and frequencydomain analyses. J Biomed Opt 2015;20:037009

36 Rizzo G, Capponi A, Arduini D, et al. The value of fetal arterial, cardiac and venous flows in predicting $\mathrm{pH}$ and blood gases measured in umbilical blood at cordocentesis in growth retarded fetuses. Br J Obstet Gynaecol 1995;102:963-9.

37 Galland BC, Taylor BJ, Bolton DP, et al. Heart rate variability and cardiac reflexes in small for gestational age infants. J App/ Physiol 2006;100:933-9.

38 Cohen E, Wong FY, Wallace EM, et al. Fetal-growth-restricted preterm infants display compromised autonomic cardiovascular control on the first postnatal day but not during infancy. Pediatr Res 2017;82:474-82.

39 Cohen J. A power primer. Psychol Bull 1992;112:155-9.

40 Alderliesten T, Lemmers PM, Smarius JJ, et al. Cerebral oxygenation, extraction, and autoregulation in very preterm infants who develop peri-intraventricular hemorrhage. J Pediatr 2013;162:698-704. 